\title{
Topology optimization of fail-safe structures using a simplified local damage model
}

\author{
Miche Jansen • Geert Lombaert - Mattias Schevenels · Ole Sigmund
}

Received: date / Accepted: date

\begin{abstract}
Topology optimization of mechanical structures often leads to efficient designs which resemble statically determinate structures. These economical structures are especially vulnerable to local loss of stiffness due to material failure. This paper therefore addresses local failure of continuum structures in topology optimization in order to design fail-safe structures which remain operable in a damaged state.

A simplified model for local failure in continuum structures is adopted in the robust approach. The complex phenomenon of local failure is modeled by removal of material stiffness in patches with a fixed shape. The damage scenarios are taken into account by means of a minimax formulation of the optimization problem which minimizes the worst case performance.

The detrimental influence of local failure on the nominal design is demonstrated in two representative examples: a cantilever beam optimized for minimum compliance
\end{abstract}

M. Jansen $(\bowtie) \cdot$ G. Lombaert

Department of Civil Engineering, KU Leuven,

Kasteelpark Arenberg 40, B-3001 Leuven, Belgium

E-mail: miche.jansen@bwk.kuleuven.be

M. Schevenels

Department of Architecture, Urbanism and Planning,

KU Leuven,

Kasteelpark Arenberg 1, B-3001 Leuven, Belgium

O. Sigmund

Department of Mechanical Engineering, Solid Mechanics,

Technical University of Denmark, Nils Koppel's Allé,

Building 404, 2800, Lyngby, Denmark

Preprint submitted to Structural and Multidisciplinary Optimization

Published version: M. Jansen, G. Lombaert, M. Schevenels and O. Sigmund. Topology optimization of fail-safe structures using a simplified local damage model. Structural and Multidisciplinary Optimization, 49:657-666, 2014. Read More: DOI 10.1007/s00158-013-1001-y and a compliant mechanism. The robust approach is applied successfully in the design of fail-safe alternatives for the structures in these examples.

Keywords Topology optimization · Robustness · Redundancy · Fail-safe design

\section{Introduction}

Topology optimization (Bendsøe and Sigmund, 2004) has become a popular tool for designing civil and mechanical structures. In many cases, the optimized design constitutes an efficient and economical solution for the application at hand, however, its topology also often resembles a statically determinate structure. Due to the lack of redundancy, these structures are very sensitive to a local failure of material. Every member in a statically determinate structure is a critical member which can not be removed without transforming the structure into an unstable mechanism and thus strongly deteriorating its performance. In civil engineering, recent work on structural safety is increasingly concerned with the consequences of local damage on the structural behavior in risk analysis (Sørensen et al., 2012; Vrouwenvelder et al., 2012). Therefore, this paper investigates how continuum topology optimization can be adopted for the design of fail-safe structures.

Two types of local failure are generally distinguished (Feng, 1988). When the loads on the structure exceed the design load, yielding of material causes local loss of stiffness or complete failure of material strength depending on the material type, i.e. ductile or brittle behavior. Failure due to material yielding is avoided by correctly dimensioning the design for the material strength and loads applicable to the structure under consideration. This paper focuses on the second type 
where local failure is generated by accidental events. In practice various incidents can cause local failure in a structure. Common sources in civil applications include corrosion, fatigue failure or calamities such as an explosion or collision. In micro-mechanical applications such as compliant mechanism, errors during the manufacturing process can also cause unexpected removal of material.

The problem of local failure and its relation to structural redundancy for truss structures has been subject of research in the past (Frangopol and Curley, 1987). Several authors (Arora et al., 1980; Feng and Moses, 1986; Marhadi et al., 2011) have also investigated the consequences of local failure on the optimal design of trusses. Marhadi and Venkataraman (2009) included nonlinear progressive failure analysis in the optimization of truss structures.

This paper is concerned with local failure in continuum topology optimization. The various scenarios of local failure are taken into account by means of a worst case formulation of the optimization problem. In a truss, local failure can be modeled straightforwardly by removing one bar from the truss. In a continuum setting, a clear distinction between structural members can not be made and local failure is manifested by cracks and voids with varying shape and size. This work, however, focuses on preventing failure of structures without actually specifying the cause of the local failure. Therefore, this complex phenomenon is approximated in a pragmatic way by considering a number of patches with a fixed shape where material is removed from the structure.

Modeling the effect of local failure poses serious challenges from a computational point of view, especially when included in a structural optimization scheme. Even the optimization of very simple trusses often forms computationally challenging problems when local failure is included. Bendsøe and Díaz (1998) and Achtziger and Bendsøe (1999) investigated problems related to damage and degradation of material properties in topology optimization of truss and continuum problems. Degradation of material is accounted for by a continuous damage field in the design domain and the worst distribution of damage is taken into account in the optimization. The parametrization of the damage is chosen such that the worst case degradation can be found by solving a convex optimization problem. The worst case consists of a uniformly distributed degradation. The present work is concerned with localized damaging of structures which does not admit such a convex formulation. Therefore, damage is limited to a small subset of the design domain where all material is removed. This enforces local removal of bars which is compensated for in the robust optimization by introducing redundant bars into the design.

Although the problems are not fully equivalent, imposing a maximum length scale on the design can alternatively increase the redundancy of the optimized structures and, in this way, increase the robustness of the designs towards local failure. Several methods have been proposed for incorporating a maximum length scale in topology optimization. Kim et al. (2004) applied a low-pass filter on the design sensitivities in order to control the member sizes. Similar to the monotonicity based minimum length scale (MOLE) method (Poulsen, 2003), a maximum length scale in the material phase can be ensured by adding local constraints to the optimization problem which impose a minimum volume of voids in every element's neighborhood (Guest, 2009). In general, however, the degree of static indeterminacy of a truss (i.e. the number of redundant bars) is a poor indicator for the overall robustness of the structure with respect to local failure (Frangopol and Curley, 1987; Sebastian, 2004; Kanno and Ben-Haim, 2011). For the same reason, the robustness of a continuum structure is not guaranteed to improve when the number of connecting members is simply increased.

This paper is organized as follows. First, topology optimization of continuum mechanical structures is recapitulated. Afterwards, the catastrophic effect of local failure on topology optimized designs is illustrated in two examples. Section 3 proposes a methodology for taking into account local material failure in topology optimization. The presented approach is employed for designing robust alternatives for the structures discussed in the previous examples. Finally, Section 4 discusses some measures for reducing the high computational cost of the presented robust optimization scheme.

\section{Deterministic topology optimization}

This paper adopts the density based approach in order to describe the material distribution in the topology optimization problem (Bendsøe and Sigmund, 2004). The design domain is discretized by means of $n_{\mathrm{e}}$ finite elements and the distribution of material is represented by a physical density $\bar{\rho}_{e}$ per element. The volume densities are allowed to vary in the interval $\bar{\rho}_{e} \in[0 ; 1]$ where 0 and 1 indicate the absence or presence of material, respectively.

The physical densities $\bar{\rho}_{e}$ are typically derived from the actual optimization variables $\rho_{e}$ by means of a density filter in order to restrict the complexity of the final design. The density filter first averages the design variables $\rho_{e}$ with a suitable weighting function $w(\mathbf{x})$ in 
order to obtain the smoothed intermediate densities $\tilde{\rho}_{e}$ (Bourdin, 2001; Bruns and Tortorelli, 2001):

$\tilde{\rho}_{e}=\frac{\sum_{i=1}^{n_{\mathrm{e}}} w\left(\mathbf{x}_{i}-\mathbf{x}_{e}\right) v_{i} \rho_{i}}{\sum_{i=1}^{n_{\mathrm{e}}} w\left(\mathbf{x}_{i}-\mathbf{x}_{e}\right) v_{i}}$

where $\mathbf{x}_{i}$ are the coordinates of the elements' centers and $v_{i}$ are the element volumes. A conic weighting function $w(\mathbf{x})=\max (R-\|\mathbf{x}\|, 0)$ with filter radius $R$ is commonly adopted. In order to remove the gray transition zones between material and void phase which result from the smoothing effect of the density filter, the intermediate variables $\tilde{\rho}_{e}$ are projected by a regularized Heaviside function (Guest et al., 2004; Wang et al., 2011):

$\bar{\rho}_{e}=\frac{\tanh (\beta \eta)+\tanh \left(\beta\left(\tilde{\rho}_{e}-\eta\right)\right)}{\tanh (\beta \eta)+\tanh (\beta(1-\eta))}$

where $\beta$ is a steepness parameter and $\eta \in[0 ; 1]$ is the threshold value of the projection. In order to improve the convergence of the optimization algorithm, the steepness parameter $\beta$ is increased during the iterations according to a continuation scheme (Sigmund, 2007).

In the optimization of linear elastic structures, the local elements' Young's moduli $E_{e}$ are expressed as function of the physical densities $\bar{\rho}_{e}$ by means of the Solid Isotropic Material with Penalization (SIMP) interpolation (Bendsøe, 1989; Zhou and Rozvany, 1991):

$E_{e}=E_{\min }+\left(E_{0}-E_{\min }\right) \bar{\rho}_{e}^{p}$

where $E_{0}$ and $E_{\text {min }}$ are the Young's moduli of the material and void phase, respectively. The penalization parameter is typically chosen $p=3$ in order to make intermediate densities inefficient in the optimization and drive the densities $\bar{\rho}_{e}$ to a discrete $\{0 ; 1\}$ design. The elements' Young's moduli $E_{e}$ are used in the construction of the global stiffness matrix $\mathrm{K}$ in the finite element analysis of the problem:

$\mathrm{K}(\boldsymbol{\rho}) \mathbf{u}(\boldsymbol{\rho})=\mathbf{f}$

where $\mathbf{u}$ and $\mathbf{f}$ are the nodal displacements and load vector, respectively, and the vector $\boldsymbol{\rho} \in \mathbb{R}^{n_{\mathrm{e}}}$ collects the design variables $\rho_{e}$.

In the following, local material failure is illustrated in the minimum compliance optimization of a cantilever beam and the design of a compliant mechanism. Both optimization problems can be formulated as follows:

$$
\begin{array}{cl}
\min _{\boldsymbol{\rho}} & f_{0}=\mathbf{b}^{\mathrm{T}} \mathbf{u}(\boldsymbol{\rho}) \\
\text { s.t. } & V(\boldsymbol{\rho})-V_{\max } \leq 0 \\
& \mathbf{0} \leq \boldsymbol{\rho} \leq \mathbf{1}
\end{array}
$$

where the volume fraction of the design $V(\boldsymbol{\rho})$ is limited to a certain fraction $V_{\max }$ of the total volume of the design domain and $\mathbf{b}$ is a problem-specific vector. The optimization problems in the examples are solved numerically by means of the Method of Moving Asymptotes (MMA) (Svanberg, 1987).

\subsection{Cantilever beam}

Local failure is first investigated in a cantilever beam optimized for minimum compliance (Fig 1(a)). The minimum compliance problem searches the stiffest structure by minimizing the work done by the external forces. This objective is obtained by setting $\mathbf{b}=\mathbf{f}$ in the optimization problem (5). A rectangular design domain is discretized by means of $60 \times 180$ unit size square finite elements. The Young's moduli of material and void phase are equal to $E_{0}=1$ and $E_{\min }=10^{-9}$, respectively. A unit load is applied in the middle of the right edge of the design domain, while the volume fraction of the design is limited to $V_{\max }=40 \%$. The density filter uses a filter radius $R=3.2$, a threshold value $\eta=0.5$ and a maximum steepness $\beta=16$ in the continuation scheme. Figure 1(b) shows the design obtained by the deterministic topology optimization. The nominal compliance of the design is equal to $f_{0}=203$.

The influence of local failure is illustrated by removing the upper left bar of the design (Fig 1(c)). In this case, the structure behaves as an unstable mechanism as shown in figures 1 (d-e) where the displacements of the nominal and damaged structures are compared. The sensitivity of the nominal design with respect to local failure is also demonstrated by the increase in compliance: when the upper bar is removed the compliance increases from $f_{0}=203$ to $f_{\max }=9166$.

\subsection{Compliant inverter}

Compliant mechanisms form a second type of mechanical structures that can be strongly influenced by local failure. For this reason, the effect of local failure on the benchmark problem of the force inverter (Sigmund, 1997) is considered. Figure 2 specifies the boundary conditions of the problem. The goal is to maximize the output displacement $u_{\text {out }}$ when the input force $f_{\text {in }}$ is applied to the mechanism. This objective is recovered by choosing the vector $\mathbf{b}$ such that the displacement component $u_{\text {out }}$ is selected from the vector $\mathbf{u}$ in optimization problem (5). The maximum volume fraction of the design is equal to $V_{\max }=25 \%$. The input force is equal to $f_{\text {in }}=2$ and the spring stiffness coefficients are $k_{\text {in }}=2$ and $k_{\text {out }}=0.002$. The design domain is 
(a)

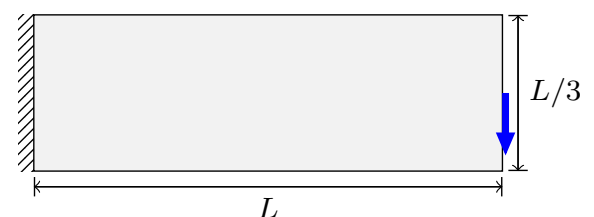

(b)
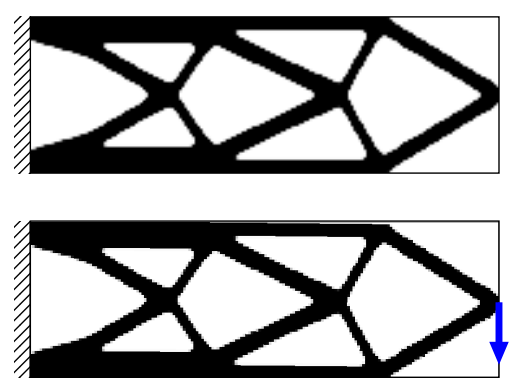

(d) (c)
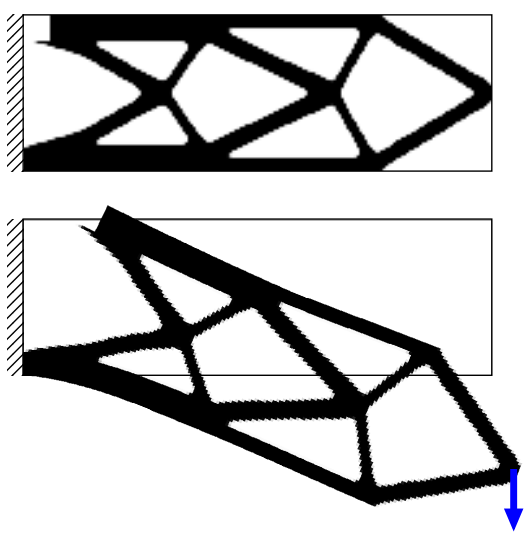

(e)

Fig. 1 (a) Design domain and boundary conditions for the cantilever problem, (b) the nominal design and (c) the design subjected to local failure of the upper bar. Figures (d) and (e) show the relative displacements of the intact and damaged structure, respectively.

(a)

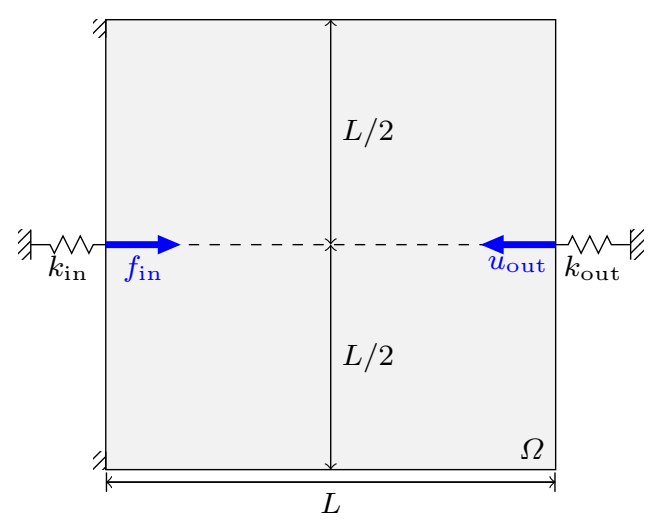

(b)
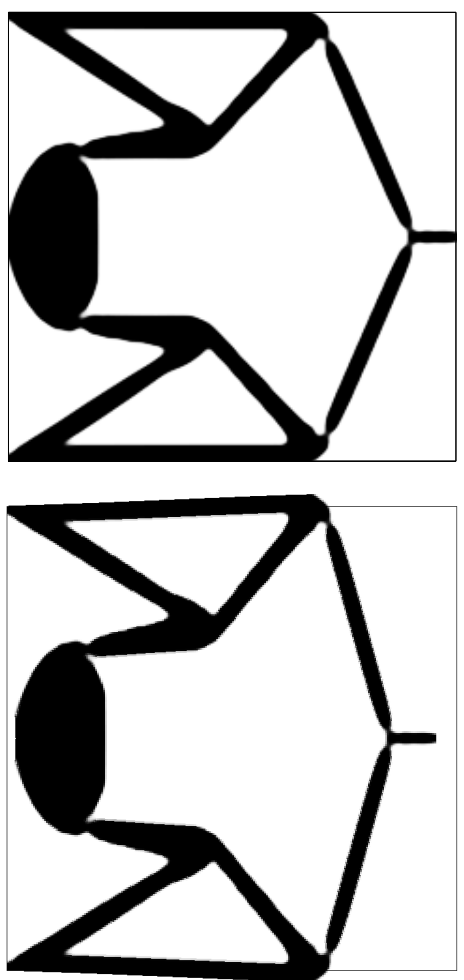

(d) (c)
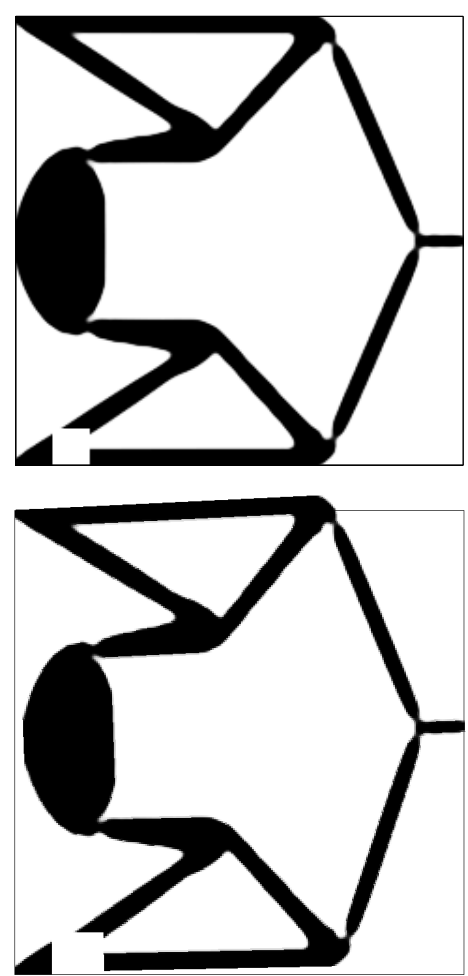

(e)

Fig. 2 (a) Design domain and boundary conditions for the compliant mechanism problem, (b) the nominal design and (c) the design exposed to local failure. Relative displacements of (d) the intact inverter and (e) the broken inverter.

discretized with $240 \times 240$ unit sized finite elements. A filter radius $R=5.6$, a threshold value $\eta=0.5$ and a maximum steepness parameter $\beta=16$ are used in the density filter. The nominal design has a performance $u_{\text {out }}=2.24$ and is shown in figure 2(b). This design is clearly very sensitive to local material removal and there are several critical parts such as the hinges in the structure where the mechanism would completely fail in 
case material would be removed locally. Figures $2(\mathrm{~d}-\mathrm{e})$, for example, compare the displacements of the nominal design and a broken design. The design is broken by local material failure close to the lower support which results in a complete loss of performance as the output displacement $u_{\text {out }}=-0.05$ becomes negative, i.e. the displacement inversion capability is lost.

\section{Local material failure}

In practice, material failure introduces cracks and holes of varying shape and size depending on factors such as the accidental event, the material type, the application and manufacturing process. A simplified model of material removal is adopted here in order to account for this complex process in the optimization problem. Furthermore, a robust approach to local failure could consist of including the worst case crack for a given volume or amount of damage in the optimization. However, the maximization problem corresponding to finding the worst case damage configuration has multiple local maxima and the solution is mesh-dependent. For example in the cantilever beam problem (Fig. 1), it is easy to see that for a limited amount of damage, a possible worst case crack would consist of very thin cracks along the height of the domain which could completely disconnect the structure from its supports. Therefore it is assumed that the damage, caused by e.g. an accident, is limited to a single location in the structure and material can only be removed in the form of a number of patches (i.e. subsets of the design domain) with a prescribed size. Since the shape of the failure mode is unknown a priori, simple rectangles will be used in the following. This patch approach can be interpreted as a model for collisions with finite-sized objects.

Local failure is modeled by changing the stiffness of the material phase $E_{0 e}$ in the SIMP interpolation (3). Let $N$ represent the index set of all elements in the design domain, then in case there is a patch of elements $P^{(i)} \subset N$ where material failure occurs, the SIMP interpolation becomes:

$E_{e}^{(i)}=E_{\min }+\left(E_{0 e}^{(i)}-E_{\min }\right) \bar{\rho}_{e}^{p}$

where $E_{0 e}^{(i)}$ is equal to:

$E_{0 e}^{(i)}= \begin{cases}E_{\mathrm{mat}} & \text { if } e \in N \backslash P^{(i)} \\ E_{\min } & \text { if } e \in P^{(i)}\end{cases}$

Figure 3 illustrates this approach for a rectangular patch.

The goal of the present work is to obtain designs which are insensitive to the occurrence of a crack or

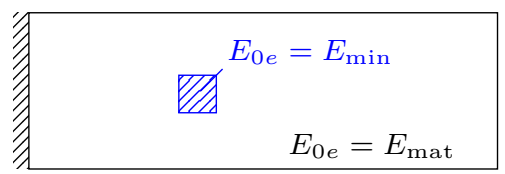

Fig. 3 Removal of a rectangular patch of material.

hole in the structure by including the failure model in the optimization. The size of the patches should be related to the expected hole size which could occur in the structure. The influence of the patch size is investigated further in the examples.

It is assumed that the uncertainty related to the occurrence of local failure can be represented by an appropriate set of $m$ patch removal scenarios. In this case, the robust optimization problem can be formulated as a scenario-based problem (Ben-Tal et al., 2009; Tempo et al., 2013) where every instance of local failure is included separately by means of a worst case formulation:

$$
\begin{array}{cl}
\min _{\boldsymbol{\rho}} & f_{\mathrm{r}}(\boldsymbol{\rho})=\max _{i=1, \ldots, m} f^{(i)}(\boldsymbol{\rho}) \\
\text { s.t. } & V(\boldsymbol{\rho})-V_{\max } \leq 0 \\
& \mathbf{0} \leq \boldsymbol{\rho} \leq \mathbf{1}
\end{array}
$$

where $f^{(i)}=\mathbf{b}^{\mathrm{T}} \mathbf{u}^{(i)}$ is the performance related to the (i)-th patch removal scenario and the displacements $\mathbf{u}^{(i)}$ solve the corresponding finite element system:

$\mathrm{K}\left(\boldsymbol{\rho} \mid E^{(i)}\right) \mathbf{u}^{(i)}(\boldsymbol{\rho})=\mathbf{f} \quad i=1, \ldots, m$

In robust optimization, the non-differentiable optimization problem (8) is typically transformed into an equivalent bound formulation by introducing a slack variable $t$ :

$$
\begin{array}{ll}
\min _{\boldsymbol{\rho}, t} & t \\
\text { s.t. } & f^{(i)}(\boldsymbol{\rho})-t \leq 0 \quad i=1, \ldots, m \\
& V(\boldsymbol{\rho})-V_{\max } \leq 0 \\
& \mathbf{0} \leq \boldsymbol{\rho} \leq \mathbf{1}
\end{array}
$$

However, if the number of scenarios $m$ is very large, the computational cost of gradient-based algorithms such as MMA increases significantly since the Jacobian of the corresponding constraint equations that is taken into account by the optimization algorithm, is a full $m \times n_{\mathrm{e}}$ matrix. Therefore, an alternative approach is followed here by approximating the nondifferentiable max-operator in the objective function by the Kreisselmeier-Steinhauser (KS) function (Kreis- 
selmeier and Steinhauser, 1983):

$$
\begin{array}{ll}
\min _{\boldsymbol{\rho}} & \tilde{f}_{\mathrm{r}}(\boldsymbol{\rho})=\log \left(\sum_{i=1}^{m} \exp \left(\gamma f^{(i)}(\boldsymbol{\rho})\right)\right) / \gamma \\
\text { s.t. } & V(\boldsymbol{\rho})-V_{\max } \leq 0 \\
& \mathbf{0} \leq \boldsymbol{\rho} \leq \mathbf{1}
\end{array}
$$

where $\gamma$ is a chosen regularization parameter in the KS function. Higher values of $\gamma$ lead to better approximations of the maximum operator, but also causes rapid variations of the gradient of $\tilde{f}_{\mathrm{r}}$ which can jeopardize the convergence of the optimization algorithm. A value between 1 and 100 for the arguments of the exponential is recommended in literature (Martins and Poon, 2005; Arnout et al., 2012). For this reason, $\gamma=50 / \hat{f}_{0}$ is adopted in the examples, where $\hat{f}_{0}$ should have the same order of magnitude as the maximum function value $f_{\mathrm{r}}$. Since the performance of the designs changes strongly during the optimization, $\hat{f}_{0}$ is updated every ten iterations and set equal to the current worst case performance. It should be emphasized that in the scenariobased formulation the complexity of the optimization problem increases with the number of patches $m$ since every damage scenario $i$ requires an additional finite element analysis. Since the location where local failure will occur in the structure is unknown in advance, it is necessary to take into account a large number of possible patches in the optimization. In order to account for all possible damage scenarios, the grid spacing of the patches is set equal to the grid spacing of the finite element mesh. Although the computational cost of this approach is very high, it ensures robustness of the solution with respect to local failure. Section 4 proposes some measures for increasing the computational efficiency.

\subsection{Cantilever beam}

The cantilever beam is optimized for three different patch sizes, $l=5, l=10$ and $l=22$, which correspond to approximately $0.23 \%, 1 \%$ and $4.5 \%$ of the volume of the design domain being affected by local failure, respectively. It is assumed that material close to the location of the load can not be removed since this would be impossible to circumvent by the structure. An inactive zone with a width equal to 20 is therefore introduced at the right hand side of domain. The spacing between the centers of neighboring patches is equal to the size of the finite elements, while patches which fall partially outside the design are not included. This leads to a total number of patches $m=8736$ for patch size $l=5, m=7701$ for $l=10$ and $m=5421$ for $l=22$.
The robust designs are shown in figure 4 . The design optimized for the patch size $l=5$ is similar to the nominal design (Fig. 1) since the patches are smaller than the width of the main bars in the nominal design. When comparing the robust designs for the patch sizes $l=10$ and $l=22$ to the nominal design, the main horizontal bars are divided into two thinner bars which improves the robustness of the designs with respect to the removal of a single bar. Furthermore, there are significant topological differences between the robust designs. The horizontal bars are separated in such a way that both bars can never be removed entirely by a single patch. The distance between these bars is therefore closely related to the size of the patches which explains the main differences between the designs in figures 4(b)(c). In this sense, the current approach also differs from a maximum length scale constraint where the distance between members is independent of the applied length scale.

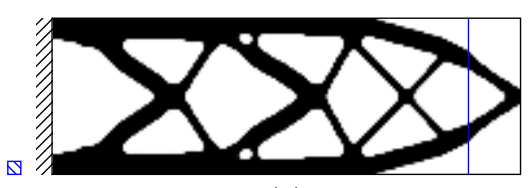

(a)

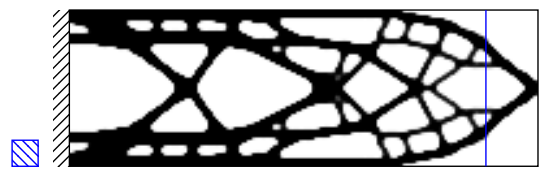

(b)

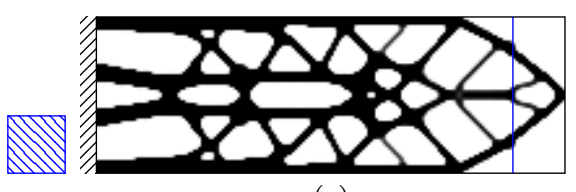

(c)

Fig. 4 Robust cantilever designs obtained for different sizes $l$ of square patches (dashed blue squares). The blue line indicates the boundary of the zone close to the location of the load where no local failure occurs.

The results for the designs are summarized in table 1 . The patch size $l=10$ for the nominal design indicates the size which was used to determine the performance $f_{\max }$ after patch removal. As expected, the robust designs have a worse nominal performance $f_{0}$ than the nominal design. Furthermore, the nominal performance $f_{0}$ increases for larger patch sizes leading to more conservative structures. Due to the increased redundancy in the robust designs, their worst case performance $f_{\max }$ is much smaller than the worst case performance $f_{\max }=9166$ of the nominal design. 
Table 1 Results for the deterministic and robust designs of the cantilever beam.

\begin{tabular}{cccccc}
\hline Design & Active patches & Figure & $l$ & $f_{0}$ & $f_{\max }$ \\
\hline Nominal & n/a & 1 & 10 & 203 & 9166 \\
\hline \multirow{4}{*}{ Robust } & all & $4(\mathrm{a})$ & 5 & 215 & 257 \\
& all & $4(\mathrm{~b})$ & 10 & 239 & 352 \\
& all & $4(\mathrm{c})$ & 22 & 304 & 714 \\
& non-empty & $8(\mathrm{a})$ & 10 & 239 & 340 \\
& non-monotonic & $8(\mathrm{~b})$ & 10 & 243 & 353 \\
\hline
\end{tabular}

With respect to the selection of an appropriate patch size, it should be noted that a design optimized for a particular patch size, will also be robust for smaller patch sizes since compliance is a monotonic function of the material stiffness. The previous results however show that overestimating the required patch size could lead to unnecessarily conservative designs.

It can be seen that the increased redundancy in the robust designs also encompasses an increased number of joints and smaller features in the design. The present failure model serves to model accidental failures at a single location in the structure and the robust optimization gives rise to designs which are more robust with respect to this specific damage model. The designs are therefore also robust to failure of a single connection or joint, but due to the presence of smaller features the design can become more vulnerable to uniform imperfections at multiple locations. The robust projection filter (Wang et al., 2011; Schevenels et al., 2011) can be adopted in case the sensitivity of the design with respect to uniform imperfections becomes important. Geometric imperfections are modeled by varying the projection threshold in the projection step (2). In the worst case approach, the robust filter additionally considers an eroded version $\overline{\boldsymbol{\rho}}^{(\mathrm{e})}=\overline{\boldsymbol{\rho}}\left(\boldsymbol{\rho} \mid \eta^{(\mathrm{e})}\right)$ and dilated version $\overline{\boldsymbol{\rho}}^{(\mathrm{d})}=\overline{\boldsymbol{\rho}}\left(\boldsymbol{\rho} \mid \eta^{(\mathrm{d})}\right)$ of the nominal design with the projection thresholds $\eta^{(\mathrm{e})}=\eta^{(\mathrm{n})}+\Delta \eta$ and $\eta^{(\mathrm{d})}=\eta^{(\mathrm{n})}-\Delta \eta$ where $\eta^{(\mathrm{n})}$ is the original nominal threshold and $\Delta \eta$ some positive shift. In general, the robust filter requires a separate finite element analysis for the three instances of the design. Due to the monotonicity of the compliance, however, it suffices to consider the eroded design in the evaluation of the objective function, i.e. the robust projection filter is incorporated by modifying optimization problem (8) as follows:

$$
\begin{array}{cl}
\min _{\boldsymbol{\rho}} & f_{\mathrm{r}}(\boldsymbol{\rho})=\max _{i=1, \ldots, m} f^{(i)}\left(\boldsymbol{\rho} \mid \eta^{(\mathrm{e})}\right) \\
\text { s.t. } & V\left(\boldsymbol{\rho} \mid \eta^{(\mathrm{n})}\right)-V_{\max } \leq 0 \\
& \mathbf{0} \leq \boldsymbol{\rho} \leq \mathbf{1}
\end{array}
$$

With compliance as the objective, incorporating the robust projection filter requires the same number of finite element analyses in the evaluation of the objective function as the original optimization problem.

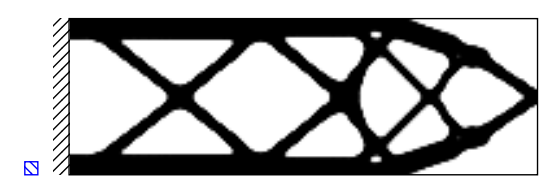

(a)

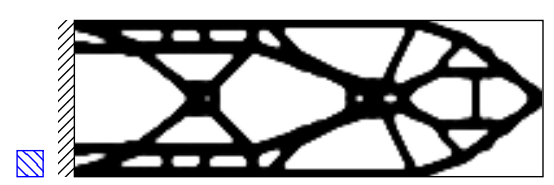

(b)

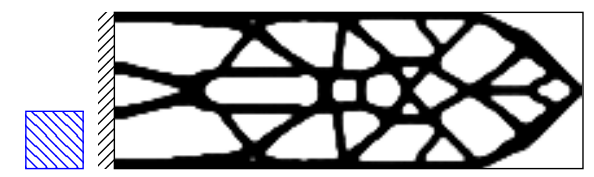

(c)

Fig. 5 Robust cantilever designs using a combined robust filter and patch removal approach with different sizes $l$ of square patches (dashed blue squares).

Figure 5 shows the designs obtained by combining the local failure model and the robust projection filter with $\Delta \eta=0.2$. The nominal performance and the performance after removing the worst case patch shown in table 2 are all higher than for the original robust designs with the same patch size in table 1 . The designs however clearly contain less joints and small features compared to the original designs in figure 4.

Table 2 Results for the robust designs of the cantilever beam optimized using different patch sizes and the robust projection filter.

\begin{tabular}{cccc}
\hline Figure & $l$ & $f_{0}$ & $f_{\max }$ \\
\hline $5(\mathrm{a})$ & 5 & 229 & 288 \\
$5(\mathrm{~b})$ & 10 & 256 & 393 \\
$5(\mathrm{c})$ & 22 & 343 & 922 \\
\hline
\end{tabular}

\subsection{Compliant inverter}

The goal of this example is to find the optimal inverter design which is robust with respect to patches with a size $l=20$. Again, two inactive zones are assumed on the left and right side of the design domain in order to avoid removal of material close to the input or output of the mechanism. The robust design is shown in 
figure 6: not only are most bars duplicated, but additional hinges appear in the design as well. Due to this increased redundancy in the robust design, the nominal performance is only $u_{\text {out }}=1.70$ compared to 2.24 for the deterministic case. However, the mechanism will keep functioning after locally removing material as the worst case performance is equal to $u_{\text {out }}=1.10$.

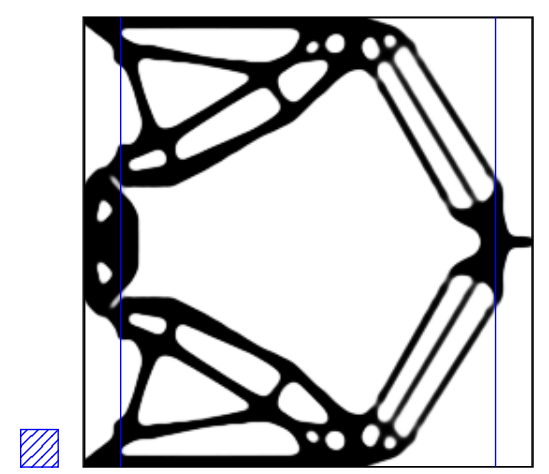

Fig. 6 The robust inverter design. The dashed blue square represents the patch size and the blue lines indicate the inactive zones close to the boundary conditions.

\section{Computational efficiency}

The aim of this paper is to show the importance of local failure in topology optimized structures and obtain designs which are fail-safe by taking into account all possible damage scenarios for a certain patch size in the optimization. Nevertheless, it is clear that the computational cost of this approach is very high due to the large number of scenarios which all require a separate finite element analysis including a factorization of a stiffness matrix K. Although the formulation of the optimization problem (11) enables a straightforward parallelization of these finite element analyses, it is necessary to investigate how the computational time of the problem could be reduced further. Several options were investigated in this respect.

Sequential formulation In every iteration of the design optimization, an topology anti-optimization could be performed by means of a gradient-based algorithm in order to find the worst case damage scenario. However, taking into account only one damage scenario in the robust optimization problem turned out to be insufficient due to the non-convexity of the maximization problem.

Perturbation techniques The effect of local failure could be taken into account in an approximate way by means of a first or second order Taylor expansion based on a sensitivity analysis for patch removal. In the probabilistic approach to robust optimization, this is often referred to as the perturbation method (Beyer and Sendhoff, 2007; Lazarov et al., 2012). Linear and quadratic approximations are adopted in a similar way in worst case approaches to robust optimization (Ma and Braatz, 2001; Diehl et al., 2006). In case of local failure, however, the damage scenarios can strongly alter the mechanical behavior of the structure which are often not predicted by first or second order sensitivities. This can be illustrated by the following physical argument. The sensitivities of the compliance with respect to a change in material stiffness are closely related to the deformation energy stored in the design (Bendsøe and Sigmund, 2004):

$$
\frac{\partial f_{0}}{\partial E_{0 e}}=-\bar{\rho}_{e}^{p} \mathbf{u}^{\mathrm{T}} \mathbf{K}_{e}^{0} \mathbf{u}
$$

where $\mathrm{K}_{e}^{0}$ is the element stiffness matrix for unitstiffness. The sensitivity with respect to stiffness changes in a patch of elements is equal to a summation of the sensitivities (13) over all elements in the patch and is therefore related to the total deformation energy stored in the patch of material.

Figure 7 shows a simple beam example originally conceived by Rozvany (2009) to illustrate the inaptitude of the deformation energy in predicting the effect of material removal: the horizontal beam is loaded by a vertical distributed force per unit length $p$ and a horizontal distributed force per unit length $2 p$. Although the deformation energy density (and hence the sensitivities) in the supporting vertical element is relatively small, it is crucial for the structural performance of the beam with a sufficiently large horizontal length $L$ under the given load. Therefore, it can be concluded that the corresponding sensitivities are also not able to indicate the worst location for local failure to occur in a structure.

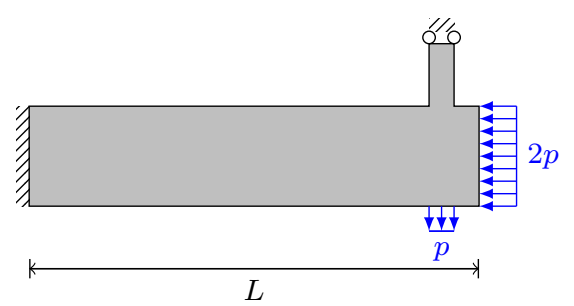

Fig. 7 Rozvany (2009) beam example where the deformation energy is not able to predict the importance of a structural element

Reanalysis techniques Akgün et al. (2001) showed that the Sherman-Morrison-Woodbury (SMW) formu- 
las provide an exact reanalysis for the displacements after a low rank modification of the stiffness matrix has occurred. This approach, however, is only computationally advantageous when the rank of the modification is small compared to the bandwidth of the stiffness matrix. This means the SHW formulas could be very useful to evaluate local failure in trusses where the removal of a bar corresponds to a rank one modification of the stiffness matrix. In the continuum setting, however, the rank of the stiffness matrix related to one patch is much larger and the computational gain is lost.

Therefore, approximate reanalysis techniques (Amir et al., 2010, 2012) are more appropriate to reduce the computational cost in this case. Amir and Sigmund (2011) used the preconditioned conjugate gradient (PCG) method to efficiently approximate the change in displacement due to patch removal. If the factorization of the stiffness matrix of the nominal design is used as a preconditioner in every iteration, only a few PCG iterations $(<10)$ are usually necessary for an accurate solution for the damaged designs. For example, following the recommendations given by Amir and Sigmund (2011), a reduction in computational time of approximately $20 \%$ was achieved in the cantilever beam problem. Relaxing the convergence criteria of the PCG method can reduce the required number of iterations and the computational time even further, however, at the risk of underestimating the effect of local failure. The interested reader is referred to Amir et al. (2012) for an extensive convergence analysis of reanalysis techniques in robust topology optimization problems and a detailed discussion on the expected computational gains.

Active set strategies The main problem of the present formulation remains the large number of active patches included in the optimization. It is clear, however, that a large fraction of these patches will not be critical during one or more iteration steps of the optimization algorithm. Therefore, an active set strategy can be developed which estimates the set of potentially critical patches in every iteration of the optimization. Two relatively conservative selection criteria based on the material distribution are investigated here. First, every patch which does not contain material, can obviously be removed from the active set of patches. Second, it can be assumed that the patches which completely cut through a bar or node of the structure will be critical. This means the active set of patches can be limited to those patches in which non-monotonic density variations occur. For every patch, the monotonicity is checked in an extended patch which consists of the patch itself plus a small layer of adjacent elements in order to ensure that some material remains when the patch is removed. The measure of monotonicity proposed by Poulsen (2003) is adopted for this purpose. The monotonicity is checked along four lines in the extended patch, i.e. the horizontal and vertical lines through the patch center and the two diagonals. Selecting the non-monotonic patches leads to a larger reduction of the number of active patches than removing the empty patches from the active set, since emptiness also implies a monotonic density variation.

Both approaches are applied to the cantilever beam for the patch size $l=10$. When the optimization is initiated with a uniform material distribution, removing the empty patches leads to reduction of the size of the active set which ranges from $0 \%$ of the total number of patches in the first steps of the optimization to approximately $10 \%$ for the final design. For the monotonicity based approach the reduction is initially $100 \%$ of the total number of patches to approximately $20 \%$ for the final design. The computational gain of the active set strategies is assessed by comparing the mean number of finite element analyses $\hat{n}_{\mathrm{FE}}$ per iteration during the first 250 iterations of the optimization algorithm. Without the application of an active set strategy the number of finite element analyses per iteration is equal to the total number of patch removal scenarios $\hat{n}_{\mathrm{FE}}=m=7701$. Removing the empty patches from the active set reduces the mean number of finite element analyses to $\hat{n}_{\mathrm{FE}}=7190$ per iteration. The monotonicity based approach leads to a stronger reduction with $\hat{n}_{\mathrm{FE}}=5874$.

The designs obtained by both approaches are shown in figure 8(a)-(b). In this example, removing the empty patches has a minor influence on the results as the topology is very similar to the original design obtained by including all patches (fig. 4(b)). The design of the monotonicity based approach 8(b) differs more strongly from the original design. The worst-case performance of both designs however is very similar to the result for the original design as shown in table 1 . The corresponding worst-case damage scenarios are shown in figures 8(c)(d). It is important to note that the worst-case patch in figure $8(\mathrm{~d})$ actually has a monotonic density variation and was therefore not present in the active set of patches during the optimization. The worst case patch in the set of non-monotonic patches results in a compliance $\tilde{f}_{\max }=352$. Due to the small difference between the estimated worst case $\tilde{f}_{\max }$ and $f_{\max }$ (Table 1), the selection of active patches based on the monotonicity criteria can still be justified for this example. It can be concluded that both strategies can successfully reduce the number of active patches in the optimization without much loss of accuracy. However, a reduction of $20 \%$ of the total number of patches will still lead to a compu- 
tationally expensive algorithm when the initial number of patches is very large (i.e. $m=6215$ compared to $m=7701$ in the present case).

\section{Conclusions}

This paper shows that topology optimized designs are often very sensitive with respect to local failure as they resemble statically determinate structures. Therefore, it is investigated how local failure can be included in the optimization problem. As opposed to truss topology optimization where a clear definition of a structural member exists, there is no real notion of a structural member in a continuum and cracks and holes of varying shape and size can occur. A simplified model of local failure is therefore used to simulate local material failure where a number of patches with predefined shape can be removed from the design.

The worst case local failure scenarios are taken into account in the robust optimization by means of a differentiable approximation of the maximum operator. The designs obtained in this way contain a number of redundant bars which leads to an increased robustness with respect to local removal of material. Although the final topologies are similar, the patch size does have a significant influence on the robust design. The results of the cantilever beam example show that a larger patch size leads to more conservative designs with a reduced nominal performance.

The present robust formulation is demanding from a computational point of view. Although parallelization of the finite element analyses and the application of reanalysis techniques are able to reduce the computational time, the problem remains relatively costly for a large number of patches.

Acknowledgements This research was supported by the NextTop project sponsored by the Villum Foundation and the KU Leuven - BOF PFV/10/002 OPTEC - Optimization in Engineering Center.

\section{References}

Achtziger W, Bendsøe M (1999) Optimal topology design of discrete structures resisting degradation effects. Structural and Multidisciplinary Optimization $17: 74-78$

Akgün M, Garcelon J, Haftka R (2001) Fast exact linear and non-linear structural reanalysis and the Sherman-Morrison-Woodbury formulas. International Journal for Numerical Methods in Engineering 50(7):1587-1606
Amir O, Sigmund O (2011) On reducing computational effort in topology optimization: how far can we go? Structural and Multidisciplinary Optimization 44:25-29

Amir O, Stolpe M, Sigmund O (2010) Efficient use of iterative solvers in nested topology optimization. Structural and Multidisciplinary Optimization 42:55-72

Amir O, Sigmund O, Lazarov BS, Schevenels M (2012) Efficient reanalysis techniques for robust topology optimization. Computer Methods in Applied Mechanics and Engineering 245246(0):217-231

Arnout S, Firl M, Bletzinger KU (2012) Parameter free shape and thickness optimisation considering stress response. Structural and Multidisciplinary Optimization 45:801-814

Arora J, Govil A, Haskell D (1980) Optimal design of large structures for damage tolerance. AIAA Journal 18:563-570

Ben-Tal A, El Ghaoui L, Nemirovski A (2009) Robust optimization. Princeton Series in Applied Mathematics, Princeton University Press

Bendsøe M (1989) Optimal shape design as a material distribution problem. Structural and Multidisciplinary Optimization 1:193-202

Bendsøe M, Díaz A (1998) A method for treating damage related criteria in optimal topology design of continuum structures. Structural and Multidisciplinary Optimization 16:108-115

Bendsøe M, Sigmund O (2004) Topology optimization: Theory, Methods and Applications, 2nd edn. Springer, Berlin

Beyer H, Sendhoff B (2007) Robust optimization - A comprehensive survey. Computer Methods in Applied Mechanics and Engineering 196(33-34):3190-3218

Bourdin B (2001) Filters in topology optimization. International Journal for Numerical Methods in Engineering 50(9):2143-2158

Bruns T, Tortorelli D (2001) Topology optimization of non-linear elastic structures and compliant mechanisms. Computer Methods in Applied Mechanics and Engineering 190(26-27):3443-3459

Diehl M, Bock H, Kostina E (2006) An approximation technique for robust nonlinear optimization. Math Program 107(1):213-230

Feng Y (1988) The theory of structural redundancy and its effect on structural design. Computers \& Structures 28(1):15-24

Feng Y, Moses F (1986) Optimum design, redundancy and reliability of structural systems. Computers \& Structures 24(2):239-251

Frangopol D, Curley J (1987) Effects of damage and redundancy on structural reliability. Journal of Struc- 


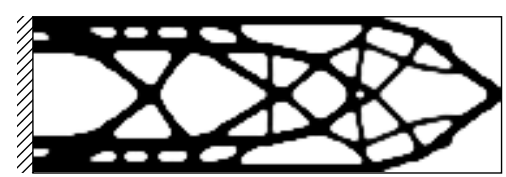

(a)

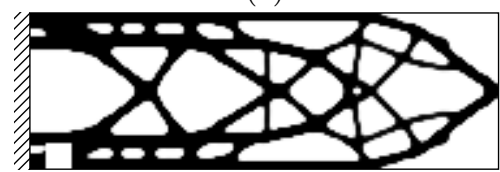

(c)

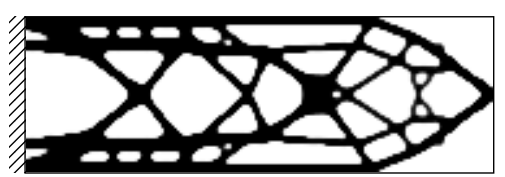

(b)

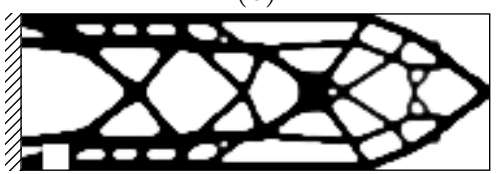

(d)

Fig. 8 Robust cantilever design obtained by (a) removing empty patches form the active set and (b) using patches which exhibit non-monotonic density variations as the active set of patches. The corresponding worst case patch removal scenarios for both designs are shown in figures (c)-(d).

tural Engineering 113(7):1533-1549

Guest J (2009) Imposing maximum length scale in topology optimization. Structural and Multidisciplinary Optimization 37:463-473

Guest J, Prevost J, Belytschko T (2004) Achieving minimum length scale in topology optimization using nodal design variables and projection functions. International Journal for Numerical Methods in Engineering 61(2):238-254

Kanno Y, Ben-Haim Y (2011) Redundancy and robustness, or when is redundancy redundant? Journal of Structural Engineering 137(9):935-945

Kim T, Kim J, Jeong J, Kim Y (2004) Filtering technique to control member size in topology design optimization. Journal of Mechanical Science and Technology 18:253-261

Kreisselmeier G, Steinhauser R (1983) Application of vector performance optimization to a robust control loop design for a fighter aircraft. International Journal of Control 37(2):251-284

Lazarov B, Schevenels M, Sigmund O (2012) Topology optimization with geometric uncertainties by perturbation techniques. International Journal for Numerical Methods in Engineering 90(11):1321-1336

Ma D, Braatz R (2001) Worst-case analysis of finitetime control policies. Control Systems Technology, IEEE Transactions on 9(5):766-774

Marhadi K, Venkataraman S (2009) Surrogate measures to optimize structures for robust and predictable progressive failure. Structural and Multidisciplinary Optimization 39:245-261

Marhadi K, Venkataraman S, Wong S (2011) Load redistribution mechanism in damage tolerant and redundant truss structure. Structural and Multidisciplinary Optimization 44:213-233

Martins J, Poon N (2005) On structural optimization using constraint aggregation. In: 6th World Congress on Structural and Multidisciplinary Optimization,
Rio de Janeiro, Brazil

Poulsen T (2003) A new scheme for imposing a minimum length scale in topology optimization. International Journal for Numerical Methods in Engineering $57(6): 741-760$

Rozvany G (2009) A critical review of established methods of structural topology optimization. Structural and Multidisciplinary Optimization 37:217-237

Schevenels M, Lazarov B, Sigmund O (2011) Robust topology optimization accounting for spatially varying manufacturing errors. Computer Methods in Applied Mechanics and Engineering 200(49-52):36133627

Sebastian W (2004) Collapse considerations and electrical analogies for statically indeterminate structures. Journal of Structural Engineering 130(10):1445-1453 Sigmund O (1997) On the design of compliant mechanisms using topology optimization. Mechanics of Structures and Machines 25(4):493-524

Sigmund O (2007) Morphology-based black and white filters for topology optimization. Structural and Multidisciplinary Optimization 33(4-5):401-424

Sørensen J, Rizzuto E, Narasimhan H, Faber M (2012) Robustness: theoretical framework. Structural Engineering International 22(1):66-72

Svanberg K (1987) The method of moving asymptotes - A new method for structural optimization. International Journal for Numerical Methods in Engineering 24:359-373

Tempo R, Calafiore G, Dabbene F (2013) Randomized Algorithms for Analysis and Control of Uncertain Systems, 2nd edn. Communications and Control Engineering, Springer-Verlag

Vrouwenvelder T, Leira B, Sykora M (2012) Modelling of hazards. Structural Engineering International 22(1):73-78

Wang F, Lazarov B, Sigmund O (2011) On projection methods, convergence and robust formulations 
in topology optimization. Structural and Multidisciplinary Optimization 43:767-784

Zhou M, Rozvany G (1991) The COC algorithm, part II: Topological, geometrical and generalized shape optimizations. Computer Methods in Applied Mechanics and Engineering 89(1-3):309-336 